\title{
Influenza y Opinión Pública en Jalisco: Un Estudio Sobre las Percepciones Ciudadanas en Guadalajara, México
}

\author{
Influenza and Public Opinion in Jalisco: \\ A study about to the public perception in Guadalajara, Mexico
}

\author{
Prof. Andrés Valdez Zepeda \\ Universidad de Nuevo México \\ azepeda@cucea.udg.mx \\ Prof. Sergio Díaz González \\ Universidad de Guadalajara \\ Profa. Delia A. Huerta Franco \\ Universidad de Guadalajara \\ Recibido: 22 de Septiembre de 2010 \\ Aceptado: 15 de Noviembre de 2010
}

\begin{abstract}
Resumen
El ensayo hace un análisis de la opinión de los jaliscienses sobre la gestión por parte del gobierno estatal de la crisis epidemiológica generada a partir de la presencia de la Influenza humana A H1N1, así como sobre el tratamiento mediático que se hizo de la misma y los posibles impactos que generó esta contingencia en la conducta de los votantes en el estado de Jalisco, México.

Se concluye que la influenza a pesar de que generó una gran cobertura informativa, convirtiéndose en un gran fenómeno mediático, mismo que estimuló y, en gran medida, moldeó la opinión pública convirtiéndola en el tema periodístico del año. Sin embargo, la agenda sanitaria y, en lo particular, la mal llamada "gripe porcina" no se ubicó como tema central en la agenda de la campaña electoral del 2009 en el estado de Jalisco, ya que los partidos políticos y sus candidatos la usaron como tema marginal. La crisis económica, la inseguridad pública, el empleo, la corrupción y la calidad y eficiencia de la gestión gubernamental fueron los temas que se impusieron en la agenda electoral, mientras que el tema de salud pública solo logró un impacto mediático coyuntural.
\end{abstract}

\section{Summary}


The essay is an analysis of the views of Jalisco on management by the state government of epidemiological crisis generated from the presence of human influenza $A H 1 N 1$, and on media treatment of it that made possible impacts generated by this contingency in the behavior of voters in the state of Jalisco, Mexico.

We conclude that the flu even though it generated a lot of coverage information, becoming a major media phenomenon, the same as stimulated and to a large extent, shaped public opinion making the subject of journalism of the year. However, the health agenda and, as particular, the poorly named "swine flu" in not placed as a central theme on the agenda of the election campaign of 2009 in the state of Jalisco, political parties and candidates used it as a marginal issue.

The economic crisis, public insecurity, employment, corruption and the quality and efficiency of government management were the topics were imposed on the electoral agenda, while the health issue public only managed a short-term media impact.

Palabras Clave: Crisis epidemiológica, influenza humana H1N1, conducta del votante, elecciones locales 2009, zona metropolitana de Guadalajara, Jalisco, México, opinión pública, fenómeno mediático, gestión de la crisis y agenda electoral.

Key Words: Crisis epidemiological human influenza H1N1, voting behavior, local elections 2009, metropolitan area of Guadalajara, Jalisco, México, public opinion, media phenomenon, crisis management and electoral agenda.

\section{Introducción}

A raíz de la aparición y propagación, en mayo del 2009, de la epidemia de Influenza humana A H1N1, también conocida como la "gripe del marrano," la cual generó una psicosis social entre los mexicanos, se consideró pertinente conocer la opinión de los jaliscienses postfact sobre la gestión de esta crisis epidemiológica por parte del gobierno del estado, el tratamiento mediático de la misma y sus posibles efectos en la conducta del votante, toda vez que la alerta epidemiológica se emitió al inicio de las campañas electorales del 2009 para renovar a los diputados federales, los diputados locales y los 125 ayuntamientos del estado de Jalisco.

Es decir, este estudio tuvo como objetivo central el indagar, tratar de entender y explicar la opinión de los jaliscienses sobre la gestión de la crisis epidemiológica por parte del gobierno, 
el tratamiento mediático que se hizo de la misma y los posibles impactos que generó la contingencia en la conducta de los votantes en el estado de Jalisco.

Conocer la opinión de los ciudadanos sobre la gestión de la epidemia nos ayudó a valorar en perspectiva las percepciones sociales sobre este tipo de acontecimientos atípicos en un contexto electoral, así como posibilita el abrevar de la experiencia jalisciense para poder gestionar, de mejor manera, acontecimientos similares en el futuro.

De igual forma, valorar la opinión de los ciudadanos sobre el tratamiento que los medios de comunicación dieron a esta contingencia epidemiológica, principalmente la televisión y la radio, permitiéndonos aquilatar, en su justa dimensión, la percepción social y su valoración sobre el trabajo informativo de los comunicadores y sus principales motivaciones.

Finalmente, es importante desde la perspectiva sociológica el tratar de conocer las percepciones sociales u opiniones ciudadanas sobre el uso político de esta emergencia por parte de los diferentes partidos políticos y el gobierno del estado para tratar de obtener un beneficio electoral.

\subsection{Preguntas de investigación e hipótesis}

En la presente investigación se trataron de encontrar respuestas a las siguientes interrogantes.

a) ¿Hubo una adecuada gestión de la contingencia epidemiológica y de la información por parte del gobierno del estado de Jalisco, de acuerdo a lo que percibió la ciudadanía?

b) ¿Cuál fue el tratamiento informativo que hicieron los medios de comunicación sobre esta contingencia sanitaria, de acuerdo a la percepción social?

c) ¿Qué efecto tuvo el surgimiento y gestión de la influenza en el comportamiento del votante de cara a las entonces elecciones locales del 5 de julio del 2009?

\subsection{Hipótesis}

A continuación se plantean tres hipótesis alternativas que tratan de dar respuesta a las preguntas de investigación. 
A1. El gobierno del estado realizó una gestión responsable de la contingencia sanitaria informando a los ciudadanos con la verdad y dando información de manera oportuna y transparente.

A2. El gobierno del estado realizó una gestión irresponsable de la contingencia sanitaria, desinformando a los ciudadanos, ocultando la verdad y, sobre todo, dando información de manera poco oportuna y transparente.

B1. Los medios de comunicación dieron un tratamiento adecuado y correcto, aquilatando adecuadamente los riesgos y peligros que representaba la influenza para la salud de las personas lo que evitó la generación de una psicosis social.

B2. Los medios de comunicación dieron un tratamiento informativo inadecuado e incorrecto, sobredimensionando los riesgos y peligros que representaba la influenza A H1N1 para la salud de las personas y generando una gran psicosis social.

C1. Los partidos políticos y sus candidatos trataron de aprovecharse de la contingencia sanitaria para lucrar políticamente y obtener un beneficio electoral.

C2. Los partidos políticos y sus candidatos no trataron de aprovecharse de la contingencia sanitaria para lucrar políticamente y de obtener un beneficio electoral.

\section{Metodología}

Para esta investigación, se levantó una encuesta aleatoria en la calle entre 430 ciudadanos de la Zona Metropolitana de Guadalajara (ZMG) entre los días 12 y 15 de mayo del 2009 para saber su opinión sobre la gestión de la influenza por parte de las autoridades gubernamentales del estado, sobre el tratamiento mediático de esta contingencia epidemiológica y sobre el posible uso de la misma por parte de los partidos y sus candidatos de cara a las elecciones del 5 de julio del 2009. ${ }^{1}$

La población de la ZMG era de 4 millones 295 mil habitantes para diciembre del 2008 . $^{2}$ Se consideró un error estadístico de $+-5 \%$ y una confiabilidad del 95\% (Scheaffer 1987 y Lohr 
2000). La selección de los entrevistados fue mediante una muestra aleatoria por conglomerados.

Se decidió realizar la encuesta en la Zona Metropolitana de Guadalajara debido a que la mayor parte de los casos de contagio y muerte producto de la influenza $\mathrm{A} \mathrm{H} 1 \mathrm{~N} 1$, así como la imposición de medidas preventivas para evitar su propagación se dieron en esta poblada zona del estado, durante las fechas del levantamiento.

\section{Marco teórico}

La opinión pública es un término polisémico, que implica diferentes conceptualizaciones, usos y procesos de formación. Por un lado, se usa para referirse a la opinión agregada de un determinado colectivo social sobre un tema en lo específico. Por ejemplo, cuando se señala que "la mayoría de la población se opone al impuesto al valor agregado (IVA) aplicable a los alimentos y las medicinas." Por el otro, se asocia a las opiniones constituidas y movilizadas de ciertos grupos de presión, quienes se movilizan en torno a un sistema de intereses explícitamente formulados (Bourdieu, 1984). ${ }^{3}$

De acuerdo a Hobbes, la opinión pública surge de la relación política del hombre con el Estado y del interés colectivo por las cuestiones de orden común. Por su parte, Rivandeneira (1976) señala que la opinión pública es, por su formación, un producto de opiniones individuales sobre asuntos de interés común y que se origina en las formas comunicativas humanas, en procesos individuales, primero y en procesos colectivos, después de diversos grados, según la naturaleza de las informaciones compartidas por los individuos, a la vez que influidas por los intereses particulares de los grupos afectados. ${ }^{4}$

Desde la perspectiva analítica, sobre este término se pueden encontrar dos modelos contrapuestos: el modelo normativo de Habermas y el modelo psicosocial de NoelleNeumann. El modelo de Habermas, enmarcada dentro del marco de la teoría crítica de la sociedad, enfatiza en las acciones comunicativas que conllevan a la construcción de la opinión pública. De cierta manera, Habermas conceptualiza a la opinión pública como un proceso racional de consenso al interior de la sociedad. Por su parte, el modelo de NoelleNeuman entiende a la opinión pública (la tiranía de la mayoría) como el conjunto de las presiones sociales básicas entendidas y sentidas por todos los individuos de una comunidad, independientemente del grado de sujeción a ellas experimentado por cada particular" (Muñoz, 1996). 
De acuerdo con la Enciclopedia Wikipedia, la opinión pública es la tendencia o preferencia real o estimulada de una sociedad hacia hechos sociales que le reportan interés.

En este trabajo, se adoptó el modelo psico-social, ya que lo que se trató de medir fue el conjunto de percepciones sobre el problema de la influenza y el tratamiento que, por un lado, le dieron los medios de comunicación y, por el otro, le otorgó el gobierno del estado y los diferentes actores. Es decir, lo que se trató de analizar fue el proceso de construcción de la opinión pública a partir de acciones, estímulos comunicativos y percepciones formadas sobre el asunto de la influenza entre un determinado colectivo social.

\section{Resultados}

De acuerdo a los resultados de la encuesta en comento, el 100\% de los entrevistados supo de la existencia de la gripe humana A H1N1. Es decir, por la forma y la dimensión de su "peligrosidad y alto nivel de contagio" con que fue comunicada por el gobierno, los diferentes medios de comunicación y los propios ciudadanos, esta pandemia logró concitar la atención de toda la sociedad.

El medio de comunicación más efectivo, que logró comunicar la existencia de esta enfermedad fue la televisión, después la radio y finalmente la prensa escrita. Según los resultados del estudio, el 75 por ciento se enteró por primera vez de la influenza por la televisión, 24 por ciento por la radio y 1 por ciento por la prensa.

Respeto de la veracidad con la que el gobierno del estado informó a los ciudadanos sobre el número de muertos y afectados por dicha pandemia, el 79 por ciento de los entrevistados consideró que si informó con la verdad y el 21 por ciento que no.

Sobre la oportuna o inoportuna intervención del gobierno del estado para evitar la propagación de esta enfermedad, el 70 por ciento de los encuestados señaló que si actuaron a tiempo, mientras que el 30 por ciento señaló que no. Casi en igual proporción, opinaron sobre la certeza de las medidas adoptadas por el gobierno del estado, ya que el 76 por ciento consideró que si tomaron las medidas adecuadas y un 24 por ciento que no.

Ahora bien, respecto al ocultamiento de la información sobre esta epidemia, la encuesta no resultó tan favorable hacia el gobierno del estado, ya que el 92 por ciento de los entrevistados señaló que si se ocultó información, mientras que el 7 por ciento opinó que no. El 1 por ciento restante no contestó. 
Sobre la calidad de la información proporcionada por el gobierno del estado, el 39 por ciento consideró que si informaron adecuadamente, un 58 por ciento señaló que sobre-informaron y el 3 por ciento que desinformaron sobre esta enfermedad.

Sobre el manejo responsable de esta crisis epidemiológica por parte del gobierno estatal, sólo el 35 por ciento consideró que si actuaron responsablemente y el 65 por ciento señaló que actuaron irresponsablemente.

Sobre el papel de los medios de comunicación en este problema, el 79 por ciento apuntó que informaron adecuada, verídica y oportunamente, mientras que el 21 por ciento de los entrevistados señaló que alarmaron innecesariamente a la población.

Ya en lo particular, sobre el uso de la epidemia para obtener beneficios colaterales por parte de los medios, el 63 por ciento consideró que se aprovecharon de la situación para obtener un mayor raiting y así aumentar las ganancias económicas, mientras que el 37 por ciento dijeron que no se aprovecharon.

Sobre el uso electoral de la epidemia, el 62 por ciento consideró que la gestión de la epidemia o la forma como se trató, si favoreció a algún partido político en lo específico (al PAN) y un 38 por ciento de los entrevistados señaló que no. El 96 por ciento de los entrevistados consideró, también, que los partidos políticos se aprovecharon de la situación para obtener una ventaja electoral, pero el 90 por ciento, señaló que el uso de este problema para tratar de ganar un mayor número de votos, no era ético. Finalmente, el 97 por ciento consideró que el tema de la salud y, en lo particular, la influenza si fue tema de las campañas electorales del 2009.

\section{Análisis e interpretación de los resultados}

Como se observó anteriormente, las percepciones sociales sobre la gestión de la influenza por parte del gobierno del estado de Jalisco y el tratamiento mediático y electoral que se hizo de esta contingencia arroja resultados contradictorios.

Por un lado, la mayoría de los ciudadanos entrevistados consideró que el gobierno del estado de Jalisco informó con la verdad, actuó oportunamente y tomó las medidas adecuadas. Por el otro lado, también consideraron que el gobierno ocultó información, sobre informó y actuó irresponsablemente. Es decir, como atinadamente, apuntara Bourdieu (1984), el proceso de formación de lo que se conoce como opinión pública es discordante, ya 
que, muchas veces, la gente no sabe lo que quiere y emite su opinión aunque no necesariamente tenga una opinión o esta sea necesariamente informada.

Esta contradicción se encontró también cuando se le cuestionó a los encuestados respecto del papel de los medios de comunicación. Por un lado, los entrevistados mayoritariamente consideraron que si informaron de manera adecuada, verídica y oportuna, pero, por el otro lado, también se aprovecharon de la situación para obtener un mayor raiting para obtener un lucro económico.

En otro orden de ideas, la mayoría de los encuestados consideró que los partidos y sus candidatos le darían un uso electoral a la influenza para obtener un lucro político y que el partido que más se beneficiaría por la presencia de la epidemia sería el PAN. Sin embargo, los resultados de las elecciones tanto estatales como nacionales favorecieron al PRI y no al PAN, como lo indicaron los entrevistados.

Es decir, una encuesta como medio para conocer la opinión pública, no necesariamente refleja lo que pasará en la realidad (ya que no son necesariamente instrumentos predictivos), sino la percepción que tiene la gente sobre un tema en lo particular, en un momento determinado. En este caso, la percepción de la mayoría de los entrevistados no coincidió con lo que finalmente pasó, ya que ni la influenza se convirtió en tema central de las campañas electorales durante el 2009, ni el PAN resultó beneficiado por la presencia de la epidemia al perder las elecciones en la mayoría de los municipios más poblados del estado de Jalisco.

\section{Conclusiones}

La presencia de la influenza A H1N1 en México, también conocida como "nueva gripe humana" fue no sólo un problema de salud pública sino, ante todo, un fenómeno mediático que alertó y, en muchos casos, atemorizó a amplios sectores de la población.

En el caso de Jalisco, la emergencia sanitaria generó una especie de psicosis social por la forma y el fondo como fue comunicada. Por un lado, tanto el gobierno federal y estatal, como los diferentes medios de comunicación sobredimensionaron, en su momento, respecto de su nivel de peligrosidad y contagio, ya que se informó que era "muy contagiosa y mortal," para la cual no había cura (vacunas).

Sin embargo, de acuerdo a la encuesta que se levantó en la ciudad de Guadalajara (México) la mayoría de los entrevistados consideró que hubo una gestión adecuada de esta 
emergencia por parte del gobierno del estado. De igual forma, la mayoría de los entrevistados señaló que los medios de comunicación dieron un tratamiento informativo adecuado, aunque si se aprovecharon de la situación para obtener un lucro económico. Finalmente, la mayoría de los encuestados consideró que se daría un uso electoral de la influenza y que su presencia beneficiaría al partido gobernante, lo cual no ocurrió, ya que el tema de salud no fue eje central del debate de las elecciones del 2009.

\section{Bibliografía}

- BOURDIEU Pierre (1984). Sociología y Cultura, México: Ed. Grijalbo/ CONACULTA.

- HABERMAS Jùrgen (1962), Historia y crítica de la opinión pública. La transformación estructural de la vida pública, Ed. Gustavo Gili, México y Barcelona, 1986.

- OCHOA, Oscar (2000). Comunicación política y opinión pública, McGrawHill/Interamericana Editores, S.A. de C.V., México D.F.

- NOËLLE NEUMANN, Elisabeth. La espiral del silencio. Opinión pública: nuestra piel social, Paidós. Barcelona, 1995

- PRICE, Vincet (1992). Opinión Pública, México: Universidad de Guadalajara.

\section{Acerca de los autores}

Andrés Valdez Zepeda. Maestro en administración pública y doctor en estudios latinoamericanos con especialidad en ciencia política por la Universidad de Nuevo México. (USA). Autor de los libros Campañas electorales inteligentes, La guerra sucia en las campañas electorales, Estrategia total en campañas electorales: estudios de casos exitosos, y El arte de ganar elecciones. Miembro del sistema nacional de investigadores. Profesor de la Universidad de Guadalajara azepeda@cucea.udg.mx

Sergio Díaz González. Maestro en ciencias de la educación en el área de planeación educativa. Profesor universitario desde 1983 en la Universidad de Guadalajara, delgado académico del CUCEA de la División de Gestión Empresarial 2002-2004 y 2004 -2006. Ha participado activamente en cuatro campañas nacionales y seis a nivel local. Actualmente, se desempeña como docente en el departamento de Administración de la Universidad de Guadalajara. 
Delia A. Huerta Franco. Tiene su maestría en administración de la educación por la Universidad de Nuevo México. Labora como asistente de investigación y es catedrática del Centro Universitario de Ciencias económico Administrativas de la Universidad de Guadalajara. Trabaja con el tema de comunicación y procesos de intercambio en el espacio público.

\footnotetext{
${ }^{1}$ De los encuestados el 62 por ciento fueron mujeres y el 38 por ciento hombres, mayores de 25 años. De esta muestra, el 20 por ciento tenía concluida sus estudios de licenciatura y 80 por ciento sin el grado de licenciatura.

${ }^{2}$ De acuerdo al Consejo Estatal de Población de Jalisco (COEPO), la población de cada uno de los municipios de dicha zona, al finalizar 2008, fue la siguiente: Guadalajara con un millón 572 mil; Zapopan, un millón 247 mil; Tlaquepaque, 617 mil; Tonalá, 451 mil; Tlajomulco, 279 mil, y El Salto, 129 mil personas.

${ }^{3}$ De hecho, Bourdieu señala que la opinión pública no existe, al menos con la forma que le atribuyen aquellos que tienen interés en afirmar su existencia."

${ }^{4}$ Rivandereira (1976). Opinión Pública, citado por Felipe Beltrán Guerrero en las memorias del evento "Comunicación y Opinión Pública: Cátedra de Opinión Pública de Carrera y en Ciencias y Técnicas de la Comunicación en la Universidad de Xalapa," en http://opinionctc.espacioblog.com/post/2005/09/aque-esopinion-publica.
}

\section{Forma de citar este artículo en bibliografías}

Valdez, Díaz y Huerta (2010): "Influenza y Opinión Pública en Jalisco", en Revista PANGEA, 1, páginas 98 a 107. Red Académica Iberoamericana de Comunicación. Recuperado el __ de de 2 de: http://revistapangea.org/2010/09/13/01-01-105/ 